\title{
Holographic Fermions in Anisotropic Einstein-Maxwell-Dilaton-Axion Theory
}

\author{
Li-Qing Fang ${ }^{1,2}$ and Xiao-Mei Kuang ${ }^{3,4}$ \\ ${ }^{1}$ School of Physics and Electronic Information, Shangrao Normal University, Shangrao 334000, China \\ ${ }^{2}$ Department of Physics and Astronomy, Shanghai Jiao Tong University, Shanghai 200240, China \\ ${ }^{3}$ Instituto de Física, Pontificia Universidad Católica de Valparaíso, Casilla 4059, Valparaíso, Chile \\ ${ }^{4}$ Department of Physics, National Technical University of Athens, 15780 Athens, Greece
}

Correspondence should be addressed to Li-Qing Fang; fangliqing@outlook.com

Received 29 November 2014; Revised 1 February 2015; Accepted 8 February 2015

Academic Editor: George Siopsis

Copyright (C) 2015 L.-Q. Fang and X.-M. Kuang. This is an open access article distributed under the Creative Commons Attribution License, which permits unrestricted use, distribution, and reproduction in any medium, provided the original work is properly cited. The publication of this article was funded by SCOAP 3 .

We investigate the properties of the holographic Fermionic system dual to an anisotropic charged black brane bulk in EinsteinMaxwell-Dilaton-Axion gravity theory. We consider the minimal coupling between the Dirac field and the gauge field in the bulk gravity theory and mainly explore the dispersion relation exponents of the Green functions of the dual Fermionic operators in the dual field theory. We find that along both the anisotropic and the isotropic directions the Fermi momentum will be effected by the anisotropy of the bulk theory. However, the anisotropy has influence on the dispersion relation which is almost linear for massless Fermions with charge $q=2$. The universal properties that the mass and the charge of the Fermi possibly correspond to nonlinear dispersion relation are also investigated.

\section{Introduction}

Landau Fermi liquid theory is a powerful tool to help physicists to understand almost all metals, like semiconductors, superconductors, and so on. However, since 1980s, plenty of features in some curious materials have been disclosed which are not well described by Landau's theory. Then non-Fermi liquid is involved in these curious materials for which the theoretical framework is still a hardcore in physics.

Recently, the AdS/CFT correspondence and more generally the gauge/gravity duality offered a possible approach to describe the non-Fermi liquid holographically. The holographic description of such a system is implemented by introducing charged fermions probe coupled to the gauge field in the gravity sector and then exploring the spectral function to disclose the Fermi surface, low energy excitations, and the type of the liquid of the dual system [1-5]. This proposal has attracted much interest and many universal features observed in laboratory have been dually described with holography. It was found that when a dipole momentum of Dirac field was introduced, the dual Fermionic system presented spectral transfer and various phases such as Fermi liquid, marginal Fermi liquid, non-Fermi liquid, and even Mott gap [6-12]. Also, the Lorentz violating boundary condition in the bulk action dual to the nonrelativistic Fermionic system which is characterized by the appearance of the flat band with gapless excitation was addressed [13].

However, in most literatures the holographic Fermionc system is dual to isotropic bulk gravity theory. Actually, the materials do not have regular structure as usual in real world. They always have different anisotropic structure [1417]. Thus, it is of great interest to holographically study a charged anisotropic bulk theory with Dirac field coupled to the gauge field. There are some works to study the anisotropic holographic fermions system. In [18], dual to isotropic bulk, the authors studied the anisotropic Fermi surface by considering BGP lagrangian [19]. The anisotropic Fermi surface with lattice was also studied in [20].

More recently, remarkable progress has been achieved in the study of anisotropic gauge/gravity dualities. Mateos and Trancanelli constructed a static and regular anisotropic black brane solution of type IIB supergravity which can be 
dually described as a spatially anisotropic $\mathcal{N}=4 \mathrm{SYM}$ plasma $[21,22]$. After that an anisotropic Einstein-MaxwellDilaton-Axion gravitational solution has been found in [23, 24]. They constructed a chareged black brane solution of type IIB supergravity with anisotropy on spacial direction, which provides a dual bulk theory to study the anisotropic holographic fermions. Some properties of the Green function of the Fermionic system dual to this kind of bulk have been studied in [25]. They found that the anisotropy of the background can lead to an anisotropic Fermi surface.

The aim of this paper is to further study the holographic Fermionic system in the charged black brane solution with anisotropy. Through calculating the Green function of the dual Fermionic operator, we will study the Fermi momentums along the anisotropic and the isotropic directions, respectively. Then we explore the critical behaviors of the low energy excitation near the Fermi surface in the two directions, respectively. We will mainly focus on the effect of the axion field parameter, the mass, and the charge of the fermions on the dispersion relation of the excitation.

The remaining of the paper is organized as follows: we will obtain the equation of motion and boundary condition in Section 2. In Section 3, we will briefly review the anisotropic Einstein-Maxwell-Dilaton-Axion theory. After that, we will solve the equations of motions numerically and disclose the low energy behavior near Fermi surface in two directions in Section 4. We present the discussion and conclusion in Section 5 .

\section{Holographic Fermionic Setup in Anisotropic Background}

In order to study the character of fermions in the dual boundary theory, we consider the bulk action for a probe Dirac fermion with the mass $m$, charge $q$. The action of bulk fermion is

$$
S_{\mathrm{bulk}}=\int d^{5} x \sqrt{-g} i \bar{\psi}(D-m) \psi
$$

and in a 5-dimensional Anisotropic black brane background

$$
d s^{2}=-g_{t t} d t^{2}+g_{r r} d r^{2}+g_{x x} d x^{2}+g_{y y}\left(d y^{2}+d z^{2}\right) \text {. }
$$

Here $\bar{\psi}=\psi^{\dagger} \Gamma^{\underline{t}}, \not D=e_{c}^{\mu} \Gamma^{c}\left(\partial_{\mu}+(1 / 4) \omega_{\mu}{ }^{a b} \Gamma_{a b}-i q A_{\mu}\right) \cdot e_{a}^{\mu}$ is the vielbein, and $\omega_{\mu}{ }^{a b}$ is the spin connection. $a, b, \ldots=\{\underline{t}, \underline{x}, \underline{y}, \underline{r}\}$ are the tangent space indices. From the above action, the Dirac equation can be written as

$$
(\not-m) \psi=0 .
$$

Then, we make a transformation $\psi=$ $\left(-g g^{r r}\right)^{-1 / 4} e^{-i \omega t+i k_{x} x+i k_{y} y+i k_{z} z} \widehat{\psi}$, where we will set $k_{z}=0$ due to the rotational symmetry of $y$ and $z$ directions, and choose the following gamma matrices basis:

$$
\begin{aligned}
& \Gamma^{\underline{r}}=\left(\begin{array}{cc}
-\sigma_{3} & 0 \\
0 & -\sigma_{3}
\end{array}\right), \\
& \Gamma^{\underline{t}}=\left(\begin{array}{cc}
i \sigma_{1} & 0 \\
0 & i \sigma_{1}
\end{array}\right), \\
& \Gamma^{\underline{x}}=\left(\begin{array}{cc}
-\sigma_{2} & 0 \\
0 & \sigma_{2}
\end{array}\right), \\
& \Gamma^{\underline{y}}=\left(\begin{array}{cc}
0 & \sigma_{2} \\
\sigma_{2} & 0
\end{array}\right) .
\end{aligned}
$$

After setting $\widehat{\psi}^{T}=\left(\widehat{\psi}_{1}, \widehat{\psi}_{2}\right)$, the Dirac equation is reduced into two coupled equations:

$$
\begin{aligned}
\sqrt{g^{r r}} \partial_{r} \widehat{\psi}_{1}+m \sigma_{3} \widehat{\psi}_{1}= & \sqrt{g^{t t}}\left(\omega+q A_{t}\right) i \sigma_{2} \widehat{\psi}_{1} \\
& -\sqrt{g^{x x}} k_{x} \sigma_{1} \widehat{\psi}_{1}+\sqrt{g^{y y}} k_{y} \sigma_{1} \widehat{\psi}_{2}, \\
\sqrt{g^{r r}} \partial_{r} \widehat{\psi}_{2}+m \sigma_{3} \widehat{\psi}_{2}= & \sqrt{g^{t t}}\left(\omega+q A_{t}\right) i \sigma_{2} \widehat{\psi}_{2} \\
& +\sqrt{g^{x x}} k_{x} \sigma_{1} \widehat{\psi}_{2}+\sqrt{g^{y y}} k_{y} \sigma_{1} \widehat{\psi}_{1} .
\end{aligned}
$$

In order to decouple the equation of motion, we set $\widehat{\psi}_{I}^{T}=$ $\left(c_{I}, d_{I}\right)$, with $I=1,2$. The equation of motion (5) yields

$$
\begin{aligned}
\sqrt{g^{r r}} \partial_{r} c_{1}+m c_{1}= & \sqrt{g^{t t}}\left(\omega+q A_{t}\right) d_{1}-\sqrt{g^{x x}} k_{x} d_{1} \\
& +\sqrt{g^{y y}} k_{y} d_{2}, \\
\sqrt{g^{r r}} \partial_{r} d_{1}-m d_{1}= & -\sqrt{g^{t t}}\left(\omega+q A_{t}\right) c_{1}-\sqrt{g^{x x}} k_{x} c_{1} \\
& +\sqrt{g^{y y}} k_{y} c_{2}, \\
\sqrt{g^{r r}} \partial_{r} c_{2}+m c_{2}= & \sqrt{g^{t t}}\left(\omega+q A_{t}\right) d_{2}+\sqrt{g^{x x}} k_{x} d_{2} \\
& +\sqrt{g^{y y}} k_{y} d_{1}, \\
\sqrt{g^{r r}} \partial_{r} d_{2}-m d_{2}= & -\sqrt{g^{t t}}\left(\omega+q A_{t}\right) c_{2}+\sqrt{g^{x x}} k_{x} c_{2} \\
& +\sqrt{g^{y y}} k_{y} c_{1} .
\end{aligned}
$$

The ingoing boundary condition for $\widehat{\psi}_{I}$ at the event horizon can be imposed by

$$
\widehat{\psi}_{I} \propto\left(\begin{array}{l}
i \\
1
\end{array}\right) e^{-i \omega r_{*}},
$$

with $r_{*}=\int\left(\sqrt{g_{r r}} / \sqrt{g_{t t}}\right) d r$. Near the AdS boundary, the solution of the Dirac equation (5) can be written as

$$
\phi_{I} \longrightarrow A_{I} r^{m}\left(\begin{array}{l}
0 \\
1
\end{array}\right)+B_{I} r^{-m}\left(\begin{array}{l}
1 \\
0
\end{array}\right) \text {. }
$$


Thus, according to AdS/CFT dictionary, the retarded Green function can be expressed as

$$
G_{I I}=B_{I}\left(A_{I}\right)^{-1}=\left(\begin{array}{ll}
G_{11} & G_{12} \\
G_{21} & G_{22}
\end{array}\right) \text {. }
$$

For simplification, in the paper we will only consider the case that the Green function is diagonal; that is, we will set $k_{y}=0$ when we study the Fermi surface along the anisotropic and set $k_{x}=0$ when we study the Fermi surface along the isotropic directions. In both of the cases, the Green function can be written as

$$
G_{I I}=\lim _{r \rightarrow \infty} r^{2 m}\left(\begin{array}{cc}
\frac{c_{1}}{d_{1}} & 0 \\
0 & \frac{c_{2}}{d_{2}}
\end{array}\right) .
$$

\section{The Anisotropic Charged Black Brane in Einstein-Maxwell-Dilaton-Axion Theory}

We start with Einstein-Maxwell-Dilaton-Axion theoretical background

$$
\begin{aligned}
d s^{2}= & e^{-(1 / 2) \phi} r^{2}\left(-\mathscr{F} \mathscr{B} d t^{2}+\mathscr{H} d x^{2}+d y^{2}+d z^{2}\right) \\
& +\frac{e^{-(1 / 2) \phi} d r^{2}}{r^{2} \mathscr{F}}, \\
A= & A_{t} d t \\
\phi= & \phi(r), \\
\chi= & a x,
\end{aligned}
$$

with the analytic solution $[23,24]$

$$
\begin{aligned}
& A_{t}=\frac{\mathscr{Q} r_{H}}{8 \sqrt{3}}\left[24\left(1-\frac{r_{H}^{2}}{r^{2}}\right)+5\left(\frac{1}{r^{2}} \ln \left(1+\frac{r_{H}^{2}}{r^{2}}\right)-\frac{\ln 2}{r_{H}^{2}}\right) a^{2}\right], \\
& \mathscr{B}=1+\frac{1}{24}\left(\frac{10 r^{2}}{\mathscr{Q}^{2} r_{H}^{4}-r^{2} r_{H}^{2}-r^{4}}+\frac{1}{r_{H}^{2} \sqrt{1+4 Q^{2}}}\right. \\
& \left.\quad \cdot \ln \left(\frac{\left(1+\sqrt{1+4 Q^{2}}\right) r_{H}^{2}+2 r^{2}}{\left(1-\sqrt{1+4 Q^{2}}\right) r_{H}^{2}+2 r^{2}}\right)\right) a^{2} \\
& +\mathscr{O}\left(a^{4}\right), \\
& \qquad(r)=-\frac{1}{4 r_{H}^{2} \sqrt{1+4 Q^{2}}}, \quad \cdot \ln \left(\frac{\left(1+\sqrt{1+4 Q^{2}}\right) r_{H}^{2}+2 r^{2}}{\left(1-\sqrt{1+4 Q^{2}}\right) r_{H}^{2}+2 r^{2}}\right) a^{2}+\mathcal{O}\left(a^{4}\right),
\end{aligned}
$$

$$
\begin{aligned}
& \mathscr{F}=1-\left(\frac{r_{H}}{r}\right)^{4}+\left[\left(\frac{r_{H}}{r}\right)^{6}-\left(\frac{r_{H}}{r}\right)^{4}\right] \mathscr{Q}^{2}+\frac{r_{H}^{4}}{24 \sqrt{1+4 Q^{2}}} \\
& \cdot\left\{3\left(-\frac{4 Q^{2}}{r^{6}}+\frac{1}{r_{H}^{6}}\right) \ln \left(\frac{\left(1+\sqrt{1+4 Q^{2}}\right) r_{H}^{2}+2 r^{2}}{\left(1-\sqrt{1+4 Q^{2}}\right) r_{H}^{2}+2 r^{2}}\right)\right. \\
& +\frac{1}{r^{2} r_{H}^{2}} \\
& \cdot\left[8 \sqrt{1+4 Q^{2}}\left(-\frac{1}{r^{2}}+\frac{1}{r_{H}^{2}}\right)\right. \\
& +\frac{1}{r^{2}}\left(3 \ln \left(-2-2 Q^{2}+2 \sqrt{1+4 Q^{2}}\right)\right. \\
& +5\left(-2+\mathbb{Q}^{2}\right) \ln \left(-1+2 \mathbb{Q}^{2}+\sqrt{1+4 Q^{2}}\right) \\
& -12 Q^{2} \ln \left(-2-2 Q^{2}+2 \sqrt{1+4 Q^{2}}\right) \\
& +7\left(1+Q^{2}\right) \\
& \cdot\left(\operatorname { l n } \left(\left(-1+2 Q^{2}-\sqrt{1+4 Q^{2}}\right)\right.\right. \\
& \cdot\left(2 Q^{2} u^{2}+\left(-1+\sqrt{1+4 Q^{2}}\right)\right) \\
& \left.\cdot\left(r_{H}^{2}\right)^{-1}\right) \\
& \left.\left.\left.\left.-\ln \left(\frac{2 Q^{2}}{r^{2}}-\frac{\left(1+\sqrt{1+4 Q^{2}}\right)}{r_{H}^{2}}\right)\right)\right)\right]\right\} \\
& \cdot a^{2}+\mathcal{O}\left(a^{4}\right)
\end{aligned}
$$

where the dimensionless charge $Q=Q / 2 \sqrt{3} r_{H}^{3}$ with $Q$ the dimensional charge corresponding to $U(1)$ gauge field. The anisotropy of the background means that the rotation symmetry is broken on the spacial direction. The anisotropic charged black brane is a solution of the equations of motion deduced from the action $[23,24]$

$$
\begin{gathered}
S=\frac{1}{2 \kappa^{2}} \int_{\mathscr{M}} \sqrt{-g}\left(\mathscr{R}+12-\frac{1}{2}(\partial \phi)^{2}-\frac{1}{2} e^{2 \phi}(\partial \chi)^{2}\right. \\
\left.-\frac{1}{4} F_{M N} F^{M N}\right) \\
+\frac{1}{2 \kappa^{2}} \int_{\partial \mathscr{M}} \sqrt{-\gamma} 2 K,
\end{gathered}
$$

with $\kappa^{2}=8 \pi G_{5}=4 \pi^{2} / N_{c}^{2} . F_{M N}$ is the strength of the $U(1)$ gauge field and $\chi$ is the axion flied. 
In Taylor series expansion of $a$, the Hawking temperature and entropy density are given by

$$
\begin{aligned}
T= & \frac{\left(2-Q^{2}\right) r_{H}}{2 \pi} \\
& +\left(-4 \sqrt{1+4 Q^{2}}+5\left(2+5 Q^{2}\right) \ln \left(\frac{3+\sqrt{1+4 Q^{2}}}{3-\sqrt{1+4 Q^{2}}}\right)\right) \\
& \cdot\left(96 \pi r_{H} \sqrt{1+4 Q^{2}}\right)^{-1} a^{2}+\mathcal{O}\left(a^{4}\right), \\
s= & \frac{N_{c}^{2} r_{H}^{3}}{2 \pi} \\
& +\frac{5 r_{H} N_{c}^{2} \ln \left(\left(3+\sqrt{1+4 Q^{2}}\right) /\left(3-\sqrt{1+4 Q^{2}}\right)\right)}{32 \pi \sqrt{1+4 Q^{2}}} a^{2} \\
& +\mathcal{O}\left(a^{4}\right) .
\end{aligned}
$$

Note that there exists thermodynamic instability of the anisotropic background. It is addressed in $[23,24]$ that the charged anisotropic background has thermodynamic instability in the region of $a^{2}>0$ and thermodynamic stability in the region of $a^{2}<0$. When $a^{2}>0$, the thermodynamic instability of the geometries solution is similar to that of the Schwarzschild-AdS with a spherical horizon. The solution corresponds to "prolate" geometry. However, the solution with $a^{2}<0$ is stable, which corresponds to "oblate" geometry. It is necessary to point out that imaginary axion field (i.e., $\left.a^{2}<0\right)$ is reasonable when we regard the axion fields as an appropriate source to support a spatially anisotropic spacetime. In the next section, we will disclose the behavior of the low energy excitation near the Fermi surface of the Fermionic system dual to both cases with $a^{2}>0$ and $a^{2}<0$.

\section{Some Properties of the Holographic Fermions}

In this section, we will set $r_{H}=1$ without loss of generality. We intent to firstly consider the massless fermions and set $q=2$. Then we will investigate the effect of mass and charge of the fermions on the dispersion relation of the Ferminic excitation. Also note that we need to fix the parameter $a$ which characterizes the level of anisotropy and the dimensionless charge $Q$. Since the temperature of our system is dependent on $a$ and $Q$, we set $Q=1.4$ in order to make sure the temperature of the system is finite and close to zero in the following discussion.

4.1. The Excitation Near Fermi Surface in Anisotropic Direction with Massless Fermions. We set $k_{y}=0$ and explore the Fermionic system on $x$-direction. We will first explore the case with $a^{2}>0$. We will numerically solve the equations of motion (6) with the use of the boundary condition (7) and read off the Green function $G_{22}$.

Our numerical result with $a^{2}=0.01$ is shown in Figure 1; the imaginary part of retarded Green function shows a peak

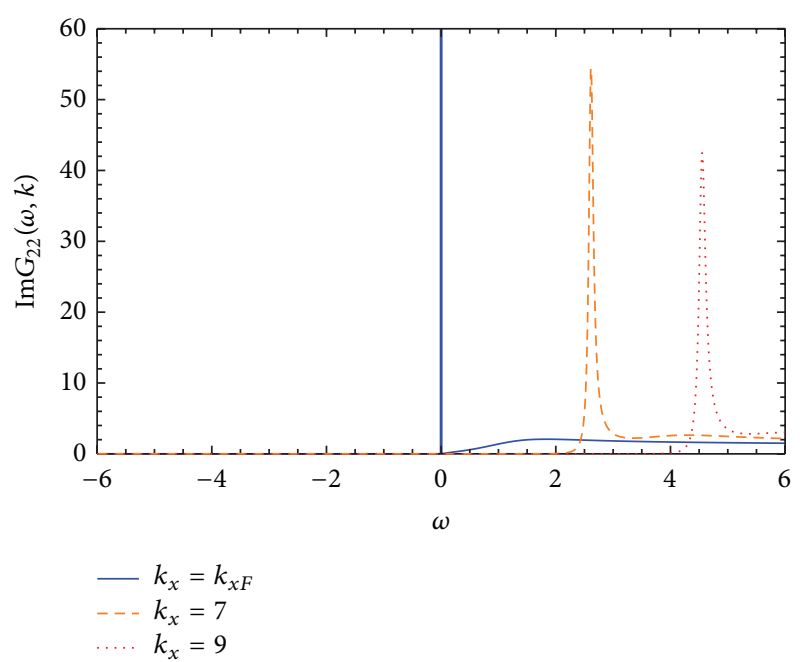

FIGURE 1: Spectral function $\operatorname{Im} G_{22}(\omega, k)$ at $k_{x}=k_{x F}$ (blue solid), $k_{x}=7$ (orange dashed), and $k_{x}=9$ (red dotted) for $a^{2}=0.01$.

in the region $\omega>0$. We see that the peak at $k_{x}=7$ is sharper than that at $k_{x}=9$. A sharp quasiparticle-like peak is generated at $\omega=0$ as the value of momentum approaches 4.2. With more careful calculation with $a^{2}=0.01$, we can determine the Fermi momentum $k_{x F}=4.18357163$ at which near zero frequency shows a peak (see Figure 2). Furthermore, we show the dependence of Fermi momentum on the degree of anisotropy in Figure 3 which shows that the Fermi momentum decreases as $a^{2}$ increases. Then, by fitting the data of $k_{x F}$ and $a^{2}$, we have

$$
k_{x F} \sim 4.20777-2.09732 a^{2} .
$$

The blue line in Figure 3 is obtained from the fitting function. When $a=0$, the Fermi momentum on anisotropic direction is just the Fermi momentum of dual liquid of 5-dimensional RN-AdS black hole.

We will move on to judge the type of liquid by studying the behavior near the Fermi surface. To achieve that, we must determine two critical exponents. One is the exponent of dispersion relation

$$
\omega_{*}\left(k_{x \perp}\right) \sim k_{x \perp}^{\alpha} \text {, }
$$

with $k_{x \perp}=k_{x}-k_{x F} \rightarrow 0_{-}$. The other is the scaling exponent of the height of $\operatorname{Im} G_{22}$ scales as $k_{x \perp}$

$$
\operatorname{Im} G_{22}\left(\omega_{*}\left(k_{x \perp}\right), k_{x \perp}\right) \sim k_{x \perp}^{-\beta} .
$$

The results of the exponents $\alpha$ and $\beta$ are shown in Figures 4 and 5 . We find that the dispersion relation for the anisotropic background almost keeps linear (the error is around $10^{-4}$ ) with the samples of small positive $a^{2}$ from Figure 4. However, the dispersion relation exponent will shift from unit if we enlarge $a$; for example, $\alpha\left(k_{x F}\right)=1.00453$ for $a=0.7$, which indicates that the parameter $a$ has imprint not only on the Fermi momentum but also on the low energy excitation near the Fermi surface. 


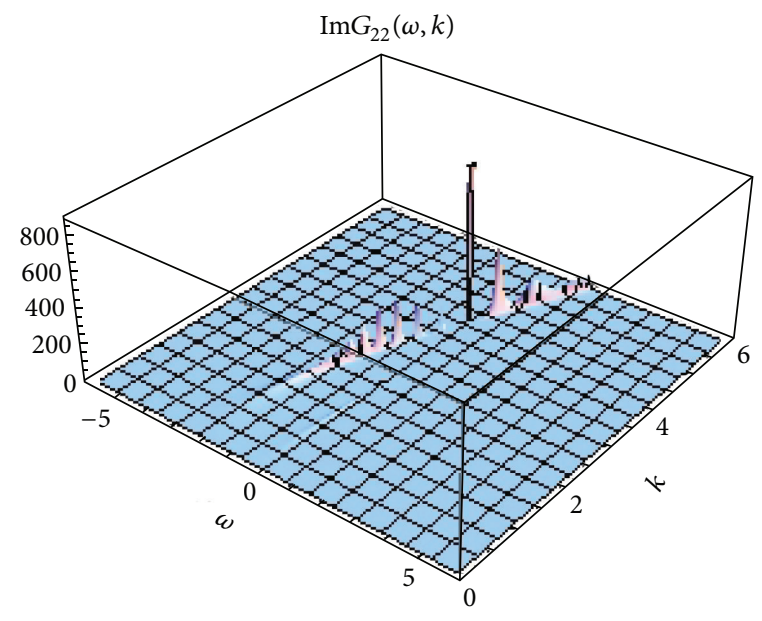

(a)

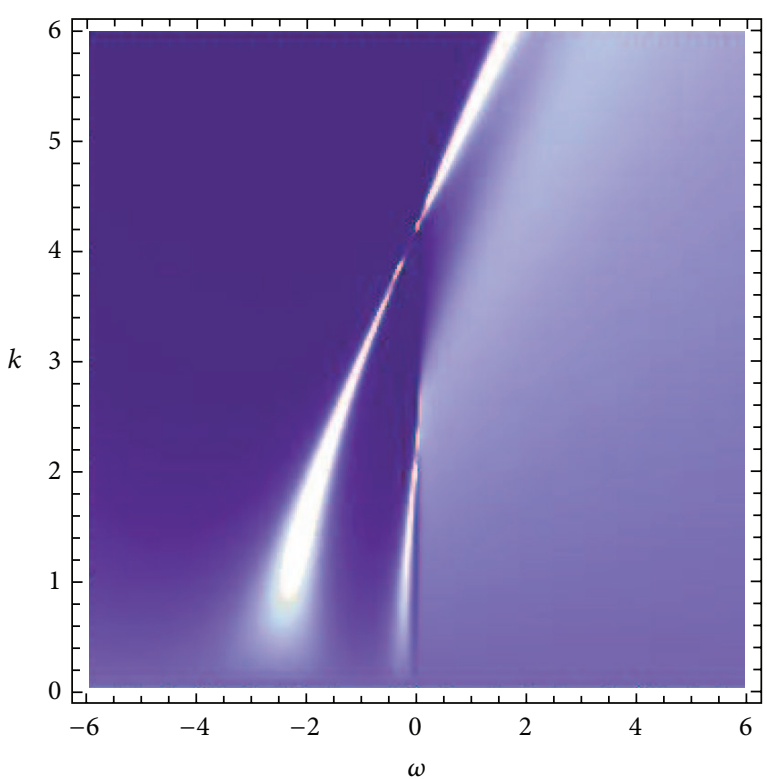

(b)

FIgUre 2: The 3D plot (a) and the density plot (b) of $\operatorname{Im} G_{22}(\omega, k)$ as $a^{2}=0.01$.

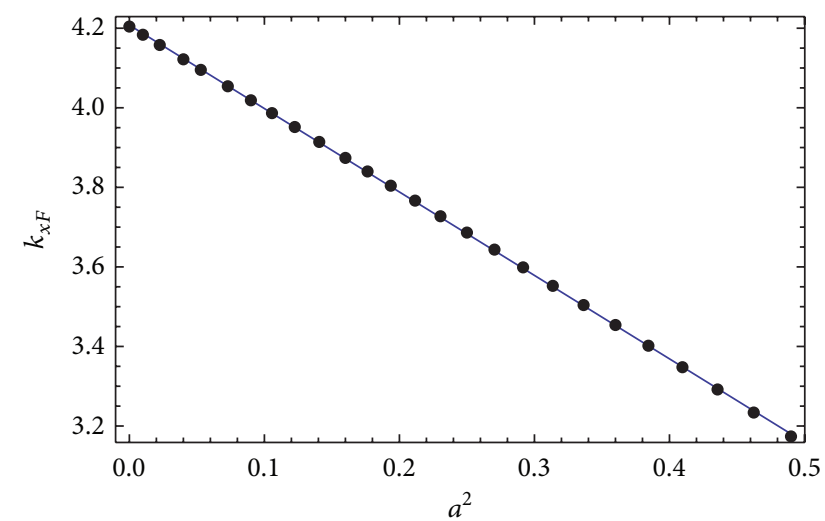

FIgURE 3: The plot of $k_{x F}$ varies as $a^{2}>0$.

In order to further check the type of the dual liquid, we must determine the other scaling relation $\beta$. For clarity, we take the logarithm of both sides of (17). After analyzing the values in Figure 5 we see that the exponent satisfies $\beta \neq 1$.

According to the statement in $[26,27]$ that the exponent of scaling behavior should obey $\alpha=\beta=1$ for Landau Fermi liquid, we claim that our dual system is not like Landau Fermi liquid system. That is to say, the system which is dual to thermodynamic instability charged axion background is nonFermi liquid-like type.

Now we turn to the background possessing thermodynamic stability with $a^{2}<0$. There appears a quasiparticle-like peak at $k_{x F}=4.224745596$ denoting the Fermi surface with $a^{2}=-0.01$. In this case we obtain that the position of Fermi surface increases as $a$ becomes smaller from Figure 6 , and the change is continuous from $a^{2}>0$ to $a^{2}<0$.
Furthermore, from Figure 7 we get that for $a^{2}=-0.01$ the scaling exponents are $\alpha \simeq 1$ and $\beta \neq 1$. We also checked that for some samples of $a^{2}<0, \alpha \simeq 1$ and $\beta \neq 1$ always can be kept, which implies that the dual liquid does not behave as Landau Fermi liquid. From the discussion above, we make a conclusion that the type of dual liquid is non-Fermi liquidlike for both stable and unstable anisotropic background.

\subsection{The Excitation Near Fermi Surface in Isotropic Direction} with Massless Fermions. In this subsection, we will set $k_{x}=$ $k_{z}=0$ to study the holographic fermions with isotropic direction. We find that as $a^{2}$ decreases, the Fermi momentum in $y$ direction increases, which is similar to that in $x$ direction. We compare the Fermi momentums in anisotropy and isotropic direction in Tables 1 and 2 . We can find that the anisotropy of the background can influence both anisotropic and isotropic direction. It is obvious that $k_{y F}$ is much smaller than $k_{x F}$ for $a^{2}>0$ while $k_{x F}$ is smaller for $a^{2}<0$.

Similarly, we also explore the scaling exponents of the low energy excitation near the Fermi surface in the $y$ direction. We find that, in isotropy direction, the scaling behaviors also satisfy $\alpha \simeq 1$ and $\beta \neq 1$ at least for small $a$, which is the same as $k_{x}$ direction. This means that the type of dual liquid in isotropic direction is also non-Fermi liquid.

4.3. The Effect of Charge and Mass of the Fermions on the Excitation. We will move on to study the influences of the charge and mass of the fermion on Fermionic excitation. Firstly, let us have a look at the influence of charge by setting $m=0$ and $a^{2}=0.01$. The influence of charge on Fermi momentums and dispersion relations with samples is shown in Table 3. As the charge of fermions is increased, the Fermi momentum becomes large and the dispersion relation 
TABLE 1: Fermi momentums $k_{x F}$ and $k_{y F}$ with samples of $a^{2}$ for $a^{2}>0$.

\begin{tabular}{lccccr}
\hline$a^{2}$ & $0.1^{2}$ & $0.08^{2}$ & $0.06^{2}$ & $0.04^{2}$ & $0.02^{2}$ \\
$k_{x F}$ & 4.18357163 & 4.19098005 & 4.19674266 & 4.20085918 & 4.20332927 \\
$k_{y F}$ & 4.18309076 & 4.19067063 & 4.1965674 & 4.20078018 & 4.20330821 \\
$k_{x F}-k_{y F}$ & 0.00048087 & 0.00030942 & 0.00017526 & 0.000079 & 0.00002106 \\
\hline
\end{tabular}

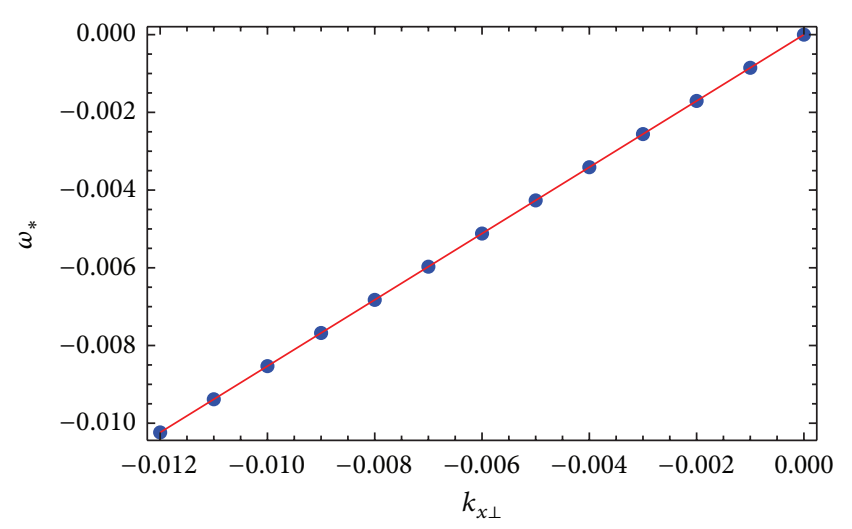

(a)

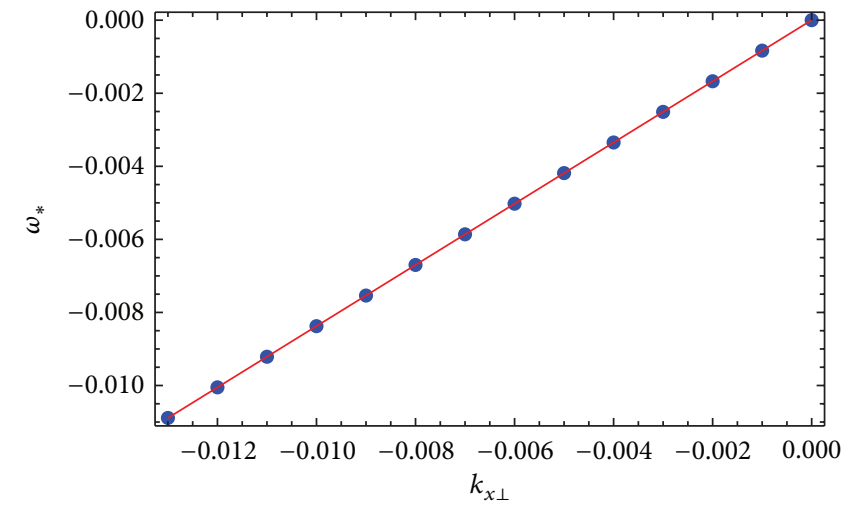

(b)

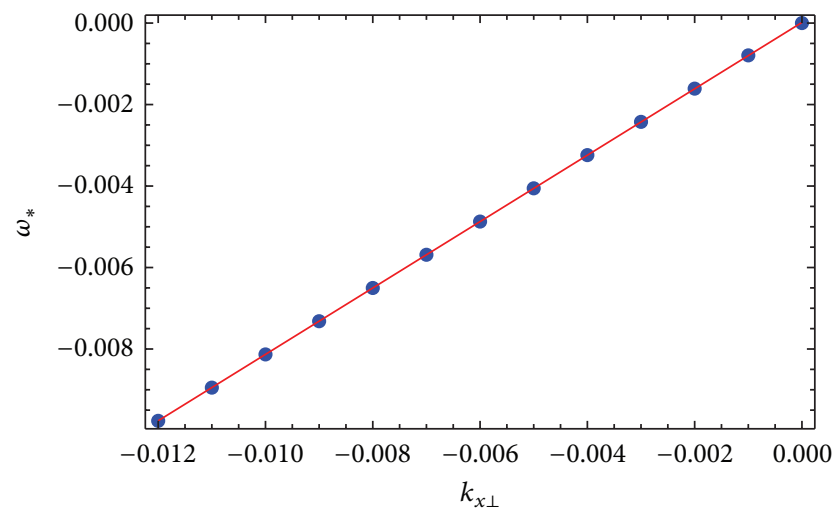

(c)

Figure 4: The dispersion relation between $k_{x \perp}$ and $\omega_{*}$ for $a^{2}=0.01(\mathrm{a}), a^{2}=0.04(\mathrm{~b})$, and $a^{2}=0.09$ (c).

approaches 1 in both isotropic and anisotropic direction. That is to say, the dual liquid is close to a Fermi liquid-like state as $q$ increases. This effect of Fermionic charge is consistent with the observation in $[2,3]$.

In Table 4, we list the Fermi momentum and exponent $\alpha$ with some samples of fermionic mass. We see that the Fermi momentum is smaller for large Fermionic mass. Also large Fermionic mass corresponds to larger $\alpha$, applying the fact that smaller mass promotes the dual liquid to appoach Fermi liquid-like state. This phenomena agrees well with that addressed in [28].

\section{Discussion and Conclusion}

In this paper, we investigated the anisotropy holographic fermions system at low temperature dual to five-dimensional Einstein-Maxwell-Dilaton-Axion gravitational background. We found that the Fermi momentum decreases as $a^{2}$ increases in both anisotropic and isotropic direction. The shape of background had effect on the value of Fermi momentum for $k_{x F}$ and $k_{y F}$. The Fermi momentum in anisotropic is larger than that in isotropic direction for "prolate" solution $\left(a^{2}>0\right)$ while the rule changes for the "oblate" solution $\left(a^{2}<0\right)$. For $a^{2}>0$ solutions, Fermi momentum gradually becomes large with the changes of the direction from the isotropic direction to anisotropic direction. Meanwhile, the Fermi momentum gradually becomes small from the isotropic direction to anisotropic direction for $a^{2}<0$ solutions. The same variation tendency towards the Fermi momentum can also be found in [25]. Finally by analyzing the dispersion relation, we discussed that the change of $a^{2}$, Fermionic charge, and mass affected the Fermi momentum and made the Fermionic system dual to Einstein-MaxwellDilaton-Axion theory deviate from Fermi liquid-like state. Because of the complexity of calculation, we only analyze the dispersion relation of the isotropic direction and anisotropic 
TABLE 2: Fermi momentums $k_{x F}$ and $k_{y F}$ with samples $a^{2}$ for $a^{2}<0$.

\begin{tabular}{lccccc}
\hline$a^{2}$ & $-0.02^{2}$ & $-0.04^{2}$ & $-0.06^{2}$ & $-0.08^{2}$ & $-0.1^{2}$ \\
$k_{x F}$ & 4.20497608 & 4.20744644 & 4.21156413 & 4.21732997 & 4.224745596 \\
$k_{y F}$ & 4.20499373 & 4.20752226 & 4.21173721 & 4.2176399 & 4.2252327 \\
$k_{x F}-k_{y F}$ & -0.00001765 & -0.00007582 & -0.00017308 & -0.00030993 & -0.000487104 \\
\hline
\end{tabular}

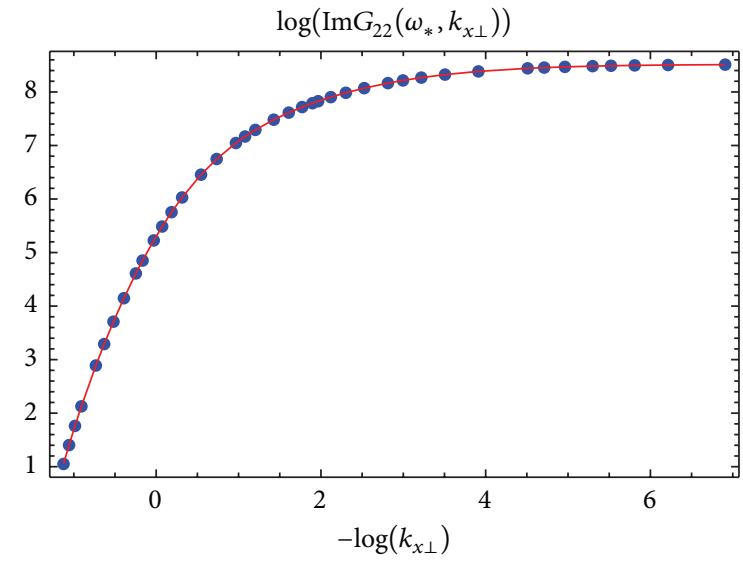

(a)

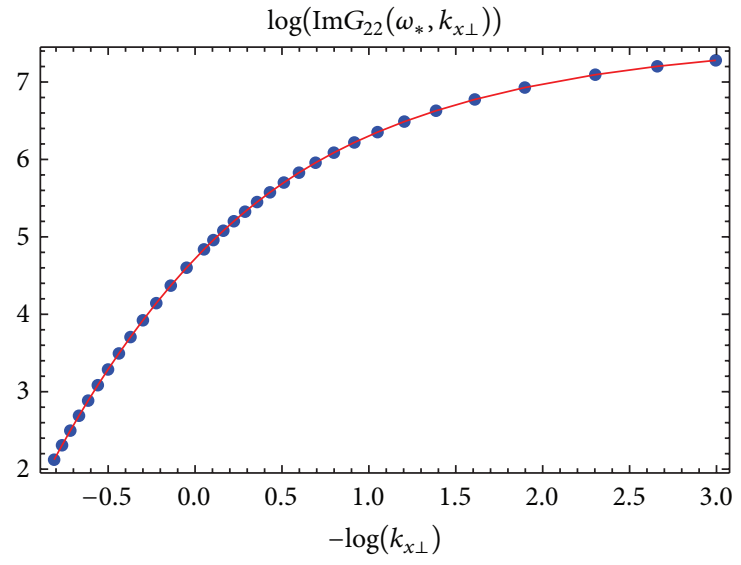

(b)

$\log \left(\operatorname{Im} G_{22}\left(\omega_{*}, k_{x \perp}\right)\right)$

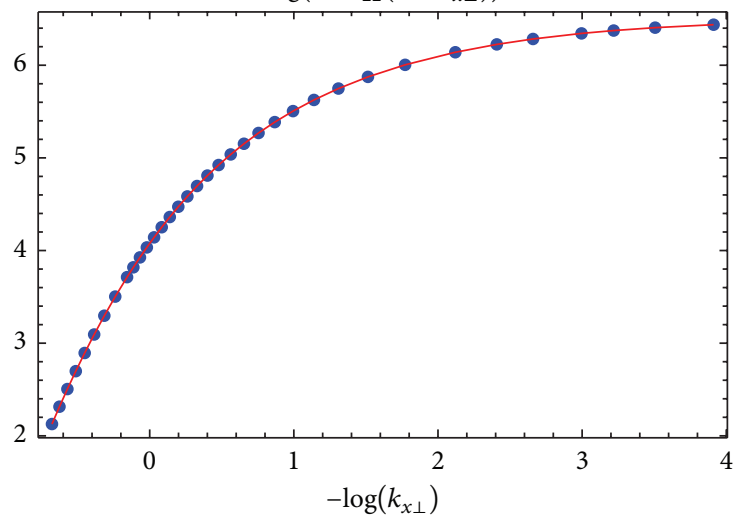

(c)

FIGURE 5: The scaling relation of the logarithm of the height of $\operatorname{Im}_{22}\left(\omega_{*}\left(k_{x \perp}\right), k_{x \perp}\right)$ at the maximum for $a^{2}=0.01(\mathrm{a}), a^{2}=0.04$ (b), and $a^{2}=0.09$ (c).

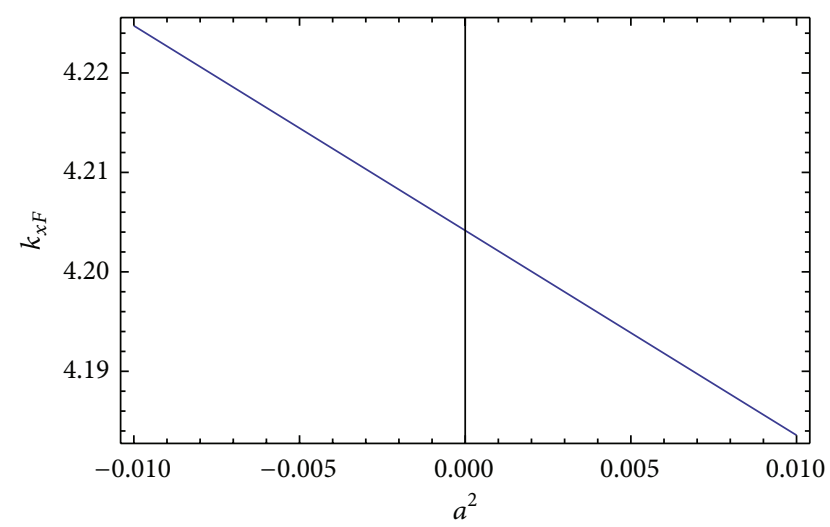

FIgURE 6: The plot of $k_{x F}$ varies as $a^{2}<0$.
TABLE 3: Fermi momentums and dispersion relation exponents with different charges for $m=0$ and $a^{2}=0.01$.

\begin{tabular}{lccc}
\hline$q$ & 0.25 & 0.5 & 2 \\
$k_{x F}$ & 0.24272478 & 0.65551085 & 4.18357163 \\
$k_{y F}$ & 0.24256306 & 0.65525147 & 4.18309076 \\
$\alpha\left(k_{x}\right)$ & 1.06664 & 1.01284 & $\approx 1$ \\
$\alpha\left(k_{y}\right)$ & 1.06876 & 1.01500 & $\approx 1$ \\
\hline
\end{tabular}

direction. The detailed analysis of the dispersion relation of more general direction will be discussed in our future work.

It is of great interest to consider the dipole coupling between Fermi and the gauge field to study the dipole effect 


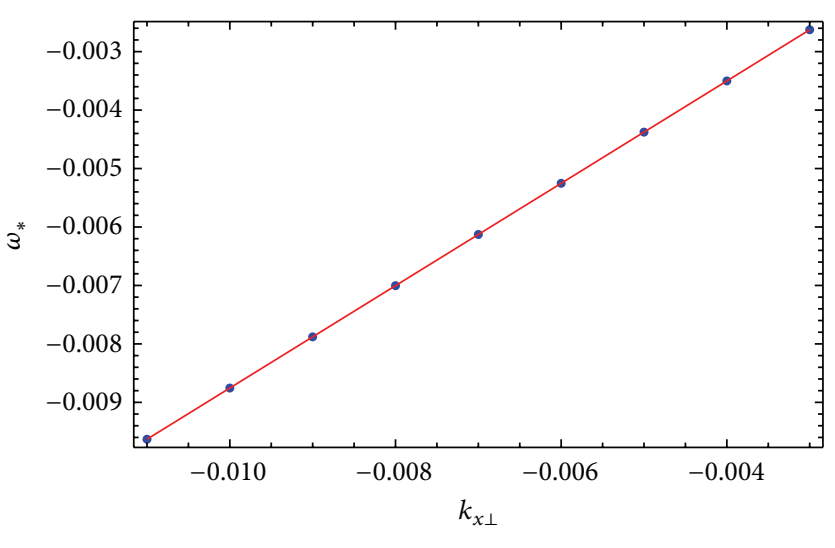

(a)

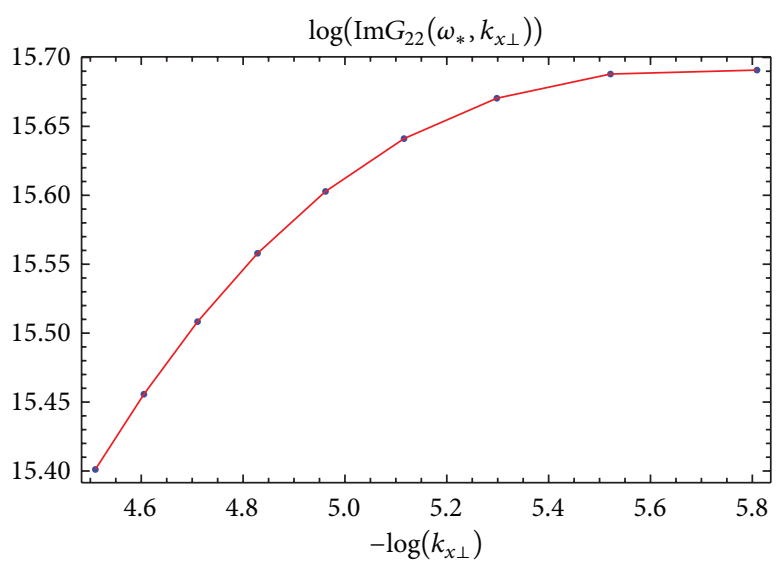

(b)

FIGURE 7: The dispersion relation (a) and the scaling behavior of $\operatorname{Im} G_{22}\left(\omega_{*}\left(k_{x \perp}\right), k_{x \perp}\right)$ (b) for $a^{2}=-0.01$.

TABLE 4: Fermi momentums and dispersion relation exponents with different masses for $q=2$ and $a^{2}=0.01$.

\begin{tabular}{lccc}
\hline$m$ & 0 & 0.1 & 0.2 \\
$k_{x F}$ & 4.18357163 & 4.05540956 & 3.92763573 \\
$k_{y F}$ & 4.18309076 & 4.05483622 & 3.79940781 \\
$\alpha\left(k_{x}\right)$ & $\approx 1$ & 1.00011 & 1.00020 \\
$\alpha\left(k_{y}\right)$ & $\approx 1$ & 1.00012 & 1.00023 \\
\hline
\end{tabular}

on the dual holographic system. Another natural direction is to turn on $k_{x}$ and $k_{y}$ to disclose the lattice structure of the Fermionic system. How to adjust our observation into the real anisotropic material is also a challenge of future work.

\section{Conflict of Interests}

The authors declare that there is no conflict of interests regarding the publication of this paper.

\section{Acknowledgments}

The authors would like to thank Long Cheng, Xian-Hui Ge, and Jian-Pin Wu for their helpful discussions. Li-Qing Fang is partly supported by Natural Science Foundation of China. Xiao-Mei Kuang is partly supported by the Chilean FONDECYT Grant no. 3150006. Xiao-Mei Kuang is also supported by ARISTEIA II action of the operational programme education and long life learning which is cofunded by the European Union (European Social Fund) and National Resources.

\section{References}

[1] S. S. Lee, "Non-Fermi liquid from a charged black hole: a critical Fermi ball," Physical Review D, vol. 79, Article ID 086006, 2009.

[2] H. Liu, J. McGreevy, and D. Vegh, "Non-Fermi liquids from holography," Physical Review D, vol. 83, no. 6, Article ID 065029, 2011.
[3] T. Faulkner, H. Liu, J. McGreevy, and D. Vegh, "Emergent quantum criticality, Fermi surfaces, and AdS2," Physical Review D, vol. 83, no. 12, Article ID 125002, 2011.

[4] M. Čubrović, J. Zaanen, and K. Schalm, "String theory, quantum phase transitions, and the emergent Fermi liquid," Science, vol. 325, no. 5939, pp. 439-444, 2009.

[5] N. Iqbal, H. Liu, and M. Mezei, "Lectures on holographic nonFermi liquids and quantum phase transitions," http://arxiv.org/ abs/1110.3814.

[6] M. Edalati, R. Leigh, and P. W. Phillips, "Dynamically generated Mott gap from holography," Physical Review Letters, vol. 106, no. 9, Article ID 091602, 4 pages, 2011.

[7] M. Edalati, R. Leigh, K. W. Lo, and P. W. Phillips, "Dynamical gap and cupratelike physics from holography," Physical Review $D$, vol. 83, Article ID 046012, 2011.

[8] W. J. Li and H. Zhang, "Holographic non-relativistic fermionic fixed point and bulk dipole coupling," Journal of High Energy Physics, vol. 2011, article 18, 2011.

[9] J. P. Wu and H. B. Zeng, "Dynamic gap from holographic fermions in charged dilaton black branes," Journal of High Energy Physics, vol. 6, article 68, 2012.

[10] W. Y. Wen and S. Y. Wu, "Dipole coupling effect of holographic fermion in charged dilatonic gravity," Physics Letters B, vol. 712, no. 3, pp. 266-271, 2012.

[11] X. M. Kuang, B. Wang, and J. P. Wu, "Dipole coupling effect of holographic fermion in the background of charged GaussBonnet AdS black hole," Journal of High Energy Physics, vol. 7, article 125, 2012.

[12] X.-M. Kuang, B. Wang, and J.-P. Wu, "Dynamical gap from holography in the charged dilaton black hole," Classical and Quantum Gravity, vol. 30, no. 14, Article ID 145011, 2013.

[13] J. N. Laia and D. Tong, "A holographic flat band," Journal of High Energy Physics, vol. 2011, article 125, 2011.

[14] S. P. Strong, D. G. Clarke, and P. W. Anderson, "Magnetic field induced confinement in strongly correlated anisotropic materials," Physical Review Letters, vol. 73, no. 7, pp. 1007-1010, 1994.

[15] S. B. Dugdale, M. A. Alam, I. Wilkinson et al., "Nesting properties and anisotropy of the Fermi surface of $\mathrm{LuNi}_{2} \mathrm{~B}_{2} \mathrm{C}$," Physical Review Letters, vol. 83, no. 23, pp. 4824-4827, 1999. 
[16] A. Vishwanath and D. Carpentier, "Two-dimensional anisotropic non-fermi-liquid phase of coupled luttinger liquids," Physical Review Letters, vol. 86, no. 4, pp. 676-679, 2001.

[17] T. Das, A. B. Vorontsov, I. Vekhter, and M. J. Graf, "Role of the Fermi surface anisotropy in angle-dependent magneticfield oscillations for identifying the energy-gap anisotropy of $\mathrm{A}_{y} \mathrm{Fe}_{2} \mathrm{Se}_{2}$ superconductors," Physical Review Letters, vol. 109, Article ID 187006, 2012.

[18] M. Edalati, K. W. Lo, and P. W. Phillips, "Pomeranchuk instability in a non-Fermi liquid from holography," Physical Review D, vol. 86, Article ID 086003, 2012.

[19] I. L. Buchbinder, D. M. Gitman, and V. D. Pershin, "Causality of massive spin-2 field in external gravity," Physics Letters. B, vol. 492, no. 1-2, pp. 161-170, 2000.

[20] Y. Ling, C. Niu, J. P. Wu, Z. Y. Xian, and H. Zhang, "Holographic fermionic liquid with lattices," Journal of High Energy Physics, vol. 2013, no. 7, article 45, 2013.

[21] D. Mateos and D. Trancanelli, "Thermodynamics and instabilities of a strongly coupled anisotropic plasma," Journal of High Energy Physics, vol. 2011, no. 7, article 54, 2011.

[22] D. Mateos and D. Trancanelli, "Anisotropic $\mathcal{N}=4$ super-Yangmills plasma and its instabilities," Physical Review Letters, vol. 107, Article ID 101601, 2011.

[23] L. Cheng, X. H. Ge, and S. J. Sin, "Anisotropic plasma with a chemical potential and scheme-independent instabilities," Physics Letters B, vol. 734, pp. 116-121, 2014.

[24] L. Cheng, X. H. Ge, and S. J. Sin, "Anisotropic plasma at finite U(1) chemical potential," Journal of High Energy Physics, vol. 2014, no. 7, article 83, 2014.

[25] L. Q. Fang, X.-H. Ge, J.-P. Wu, and H.-Q. Leng, "Anisotropic Fermi surface from holography," http://arxiv.org/abs/1409.6062.

[26] T. Senthil, "Critical Fermi surfaces and non-Fermi liquid metals," Physical Review B, vol. 78, no. 3, Article ID 035103, 14 pages, 2008.

[27] T. Senthil, "Theory of a continuous Mott transition in two dimensions," Physical Review B, vol. 78, Article ID 045109, 2008.

[28] X. M. Kuang, E. Papantonopoulos, B. Wang, and J. P. Wu, "Formation of Fermi surfaces and the appearance of liquid phases in holographic theories with hyperscaling violation," Journal of High Energy Physics, vol. 2014, no. 11, article 86, 2014. 

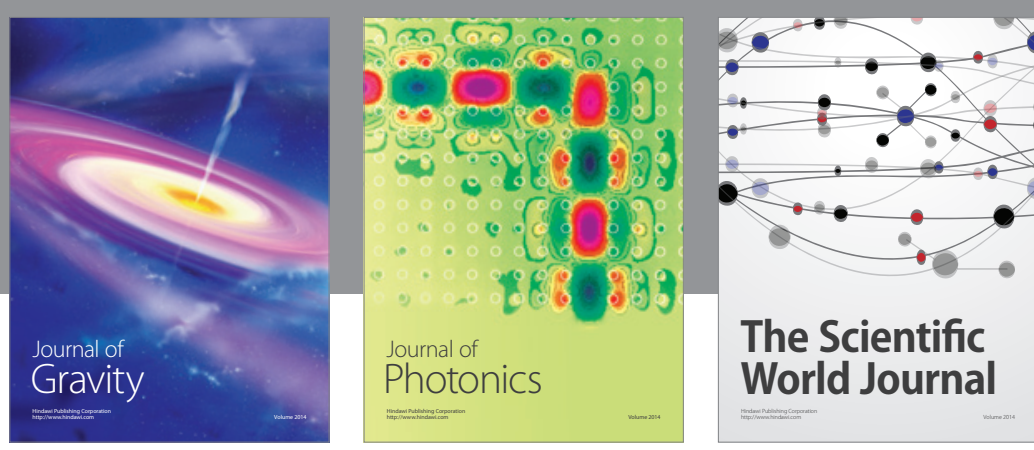

The Scientific World Journal
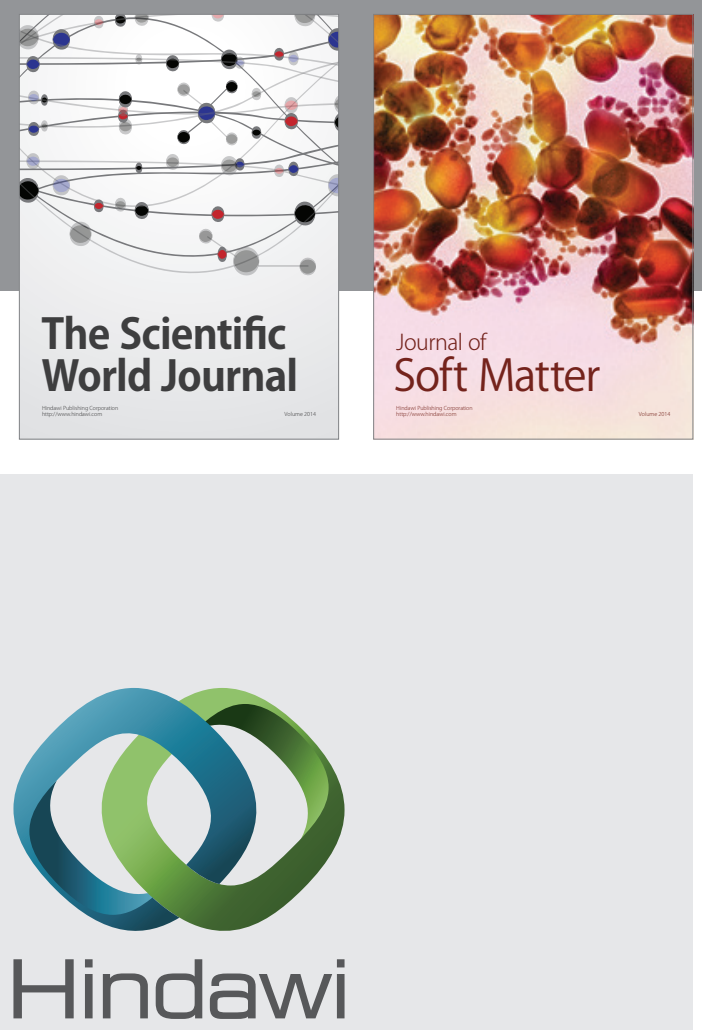

Submit your manuscripts at

http://www.hindawi.com

nternational Journal of

Statistical Mechanics
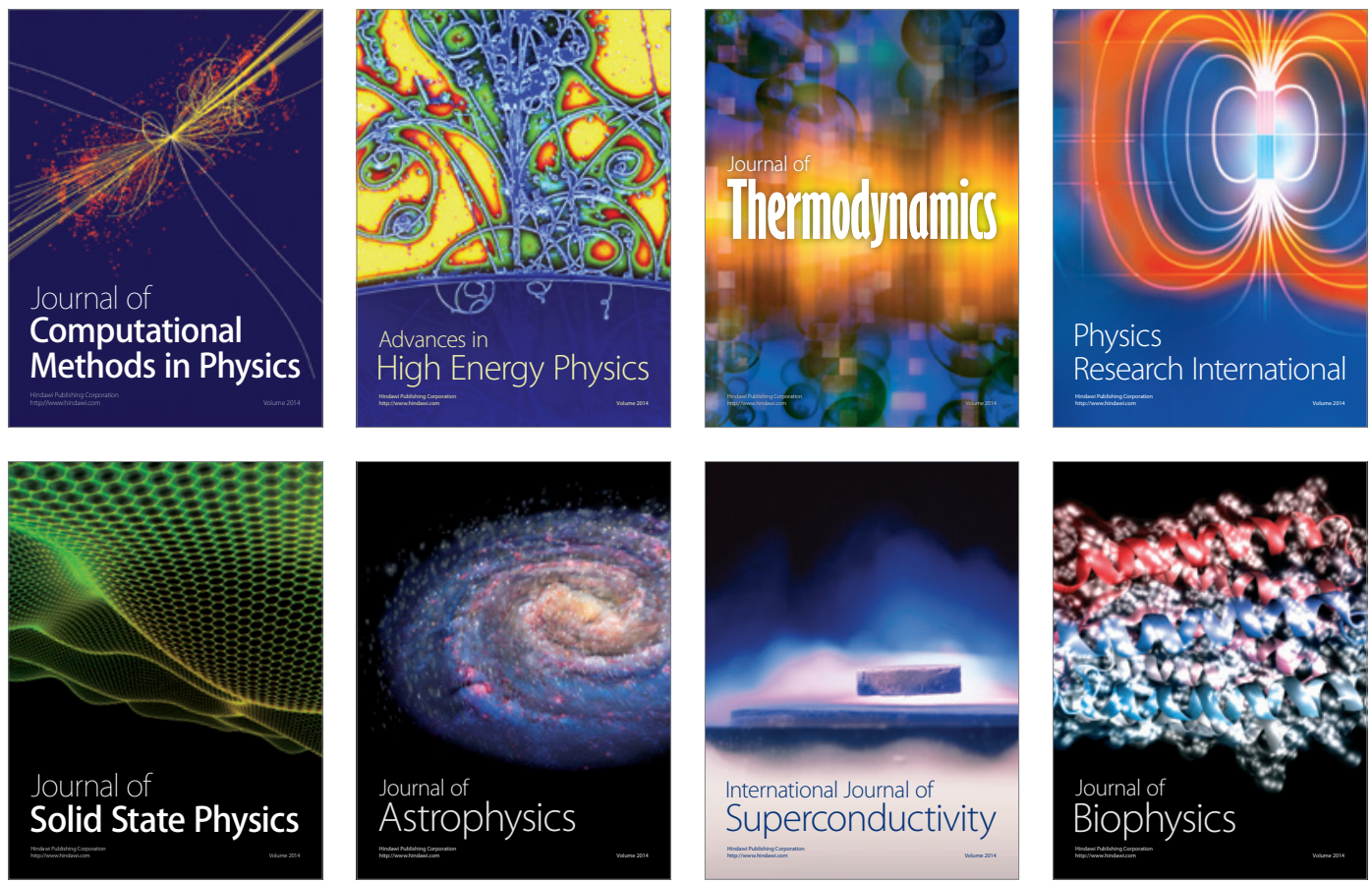
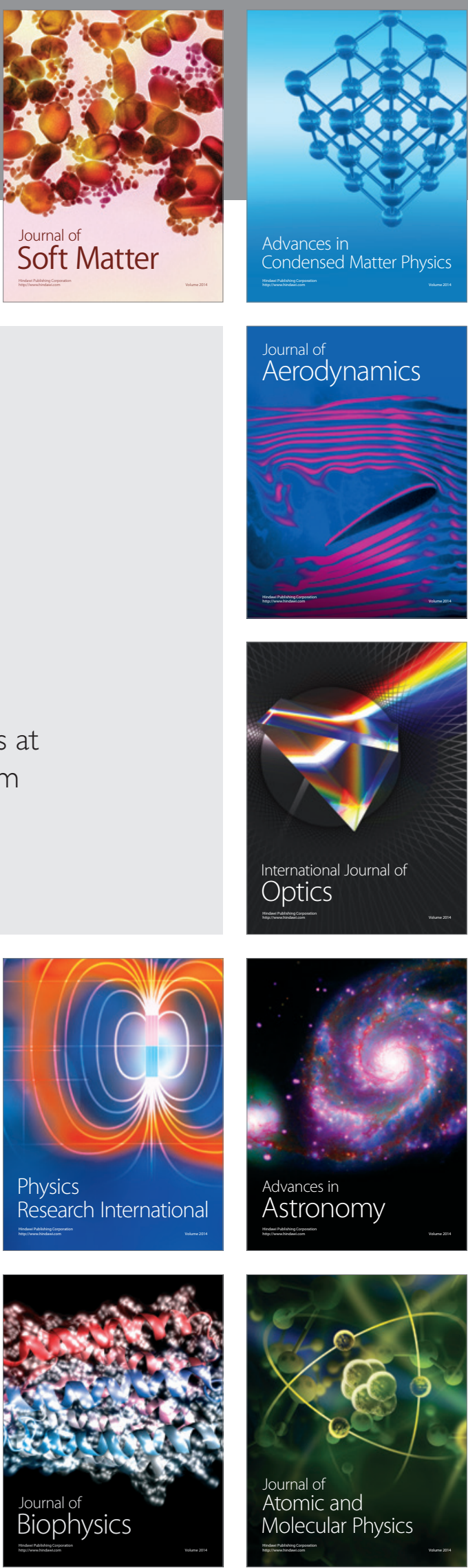